\title{
Systemic Effects of Selection History on Learned Ignoring
}

5

6

7

8

9

10

11 To whom correspondence should be addressed:

12 Andy Jeesu Kim

13 University of Southern California

14 Department of Gerontology

153715 McClintock Ave

16 Los Angeles, CA 90089

17 andyk@usc.edu

18

19

20

21

22

23

24

25

26
Andy J. Kim* \& Brian A. Anderson

Texas A\&M University

*Andy Jeesu Kim is now at the University of Southern California

*Manuscript is accepted at Psychonomic Bulletin \& Review, December 2021 
Abstract

Despite our best intentions, physically salient but entirely task-irrelevant stimuli can sometimes capture our attention. With learning, it is possible to more efficiently ignore such stimuli, although specifically how the visual system accomplishes this remains to be clarified. Using a sample of young-adult participants, we examined the time course of eye movements to targets

32 and distractors. We replicate a reduced frequency of eye movements to the distractor when

33 appearing in a location at which distractors are frequently encountered. This reduction was

34 observed even for the earliest saccades, when selection tends to be most stimulus-driven.

35 When the distractor appeared at the high-probability location, saccadic reaction time was

36 slowed specifically for distractor-going saccades, suggesting a slowing of priority accumulation

37 at this location. In the event that the distractor was fixated, disengagement from the distractor 38 was also faster when it appeared in the high-probability location. Both proactive and reactive 39 mechanisms of distractor suppression work together to minimize attentional capture by 40 frequently-encountered distractors.

41

42 Key words: selective attention, attentional capture, selection history, signal suppression, eye 43 movements 
45 Distraction can have serious health, occupational, and educational consequences. When a

46 specific distracting event is experienced frequently, individuals can learn to better ignore it.

47 Specifically what it is that changes about how a person directs their attention in order to

48 facilitate this ignoring is not well understood. In the present study, we demonstrate a

49 widespread influence of learning on the ability to resist distraction across multiple aspects of

50 attentional control. Our findings reveal that learning to ignore is a systemic process, which

51 efforts to facilitate learned ignoring should take into account. 
Although we are capable of focusing our attention on stimuli that are relevant to our

53 current goals and needs (e.g., Kiss et al., 2009; Wolfe \& Horowitz, 2017), we can at times be

54 distracted by stimuli that we know to be task-irrelevant even when we try our best to ignore them. Certain stimuli are more likely than others to break our focus and capture our attention.

56 Such stimuli include previously reward-associated (e.g., Anderson et al., 2011) and aversively-

57 conditioned stimuli (e.g., Schmidt et al., 2015; Anderson \& Britton, 2020). Physically salient stimuli have long been observed to function as potent distractors (see Theeuwes, 2010, for a

59 review), with a well-characterized time course in which the attentional priority of such stimuli is

60 initially high and then dissipates over time as the priority of the task-relevant target increases,

61 reflecting the more sluggish influence of task goals on biased competition (e.g., Donk \& van

62 Zoest, 2008; Godjin \& Theeuwes, 2002; van Zoest et al., 2004; van Zoest \& Donk, 2005).

63 Distraction by task-irrelevant stimuli can have significant health, occupational, and educational consequences (e.g., Namian et al., 2018; Strayer \& Drews, 2004; Taneja et al., 2015), and so it is

65 important to understand the mechanisms by which distraction can be mitigated.

Under certain conditions, the processing of physically salient stimuli can be suppressed

67 (e.g., Gaspelin et al., 2015, 2017; Geng \& Diquattro, 2010), a process believed to play a key role in the control of attention (Luck et al., 2021). Recent research demonstrates a powerful role for

69 learning in the ability to resist distraction via suppressive mechanisms. When a physically

70 salient but task-irrelevant distractor is more likely to appear at a particular location in space,

71 attentional capture by that distractor is substantially reduced when appearing in this high-

72 probability location (e.g., Wang \& Theeuwes, 2018a, 2018b, 2018c). This spatially-specific 
73 reduction in capture persists once the biased spatial probabilities are removed (Britton \&

74 Anderson, 2020; Kim \& Anderson, 2021), implicating learning-dependent processes. A similar

75 reduction in the magnitude of attentional capture by physically salient stimuli has been

76 observed as a function of the frequency with which distractors appear in a particular color

77 (Stilwell et al., 2019; Vatterott \& Vecera, 2012; see also Failing et al., 2019).

Specifically how the visual system implements such statistical learning in the mitigation

79 of distraction remains to be clarified, including whether a single or multiple mechanisms of

80 distractor suppression are implicated and the manner in which they are leveraged to modulate

81 information processing. Both proactive and reactive mechanisms of attentional control

82 contribute to the mitigation of distraction, reconfiguring how visual information will be

83 processed in advance of seeing a display and quickly adjusting based on what is detected in the

84 environment, respectively (see Geng, 2014). One study related statistically-learned distractor

85 suppression to pre-trial neural oscillations in the alpha band as measured using

86 electroencephalography (EEG), suggesting a proactive mechanism of suppression (Wang et al.,

87 2019b). The time course of this putatively proactive influence of suppression on stimulus

88 selection, and whether it can mitigate capture even at the earliest stages of competition within

89 the attention system, is not known. Mechanisms of reactive attentional control could also be

90 subject to learning from statistical regularities and assist with the mitigation of attentional

91 capture by frequent distractors, although such mechanisms have received little consideration in

92 the emerging literature linking distractor suppression to statistical learning. 
To gain additional insights into these issues, we examined the time course of

statistically-learned distractor suppression as a function of saccadic reaction time (sRT) in an oculomotor attentional capture task. Statistical learning of a high-probability distractor location has been shown to modulate the frequency of distractor and target fixations, with selection more strongly favoring the target when the distractor appears in the high-probability location (Wang et al., 2019a). Leveraging the well-characterized time course of eye movements to physically salient distractors and targets as a function of sRT (Donk \& van Zoest, 2008; Godjin \& Theeuwes, 2002; van Zoest et al., 2004; van Zoest \& Donk, 2005), we looked for evidence for proactive and reactive mechanisms of mitigating distraction tied to statistical learning.

Proactive attentional control would be evident in a reduced frequency of distractor fixations for distractors appearing at the high-probability location even for the most rapidly triggered eye movements, reflecting an influence of statistical learning on the most stimulus-driven saccades. Relatedly, a slowing of SRT for distractor-going saccades would be consistent with a slowing of priority accumulation at this location. Distinctly reactive attentional control would be evident in speeded disengagement from a distractor when appearing at the high-probability location (Wang et al., 2019a).

\section{Methods}

\section{Participants}

Thirty-six participants ( 26 female), between the ages of 18 and 33 inclusive $(M=20.5$, $S D=3.0)$, were recruited from the local University community. All participants were Englishspeaking and reported normal or corrected-to-normal visual acuity and normal color vision. All 
114 procedures were approved by the University Institutional Review Board and were conducted in

115 accordance with the principles expressed in the Declaration of Helsinki. Written informed

116 consent was obtained for each participant. Our sample size was based off a power analysis

117 (G*Power 3.1, $\alpha=0.05,1-\beta>0.8)$. From Wang et al. (2019a), the effect size $d_{z}(t / \operatorname{sqr}(\mathrm{n}))$ for the

118 difference in oculomotor capture for distractors appearing in the high- vs. low-probability

119 distractor locations was $d_{z}=1.493$; for (manual) response time (RT) it was $d_{z}=1.765$ and for

120 oculomotor dwell time it was $d_{z}=1.305$. Since the effect size for oculomotor capture in smaller

121 bins of trials (based on SRT) may be somewhat reduced, we powered our study to detect an

122 effect with power $(1-\beta)>0.8$ as small as $d_{z}=0.481$, which is less than one-third the size of the

123 effect over all trials reported in Wang et al. (2019a). Our sample provided power (1- $\beta$ ) $>0.98$ to

124 replicate even the smallest pairwise comparison reported as significant in Wang et al. (2019a).

125 Apparatus

126

A Dell OptiPlex 7040 (Dell, Round Rock, TX, USA) equipped with Matlab software

127 (Mathworks, Natick, MA, USA) and Psychophysics Toolbox extensions (Brainard, 1997) was used

128 to present the stimuli on a Dell $\mathrm{P} 2717 \mathrm{H}$ monitor. The participants viewed the monitor from a

129 distance of approximately $70 \mathrm{~cm}$ in a dimly lit room. Eye-tracking was conducted using the

130 EyeLink 1000 Plus system (SR Research Ltd., Ottawa, Ontario, Canada), and head position was

131 maintained using a manufacturer-provided chin rest (SR Research Ltd.).

\section{Stimuli and Task}

Each trial consisted of a gaze-contingent fixation display, a visual search array, and an

134 inter-trial-interval (ITI; see Figure 1). The fixation display consisted of a fixation cross $\left(0.7^{\circ} \times 0.7^{\circ}\right.$ 


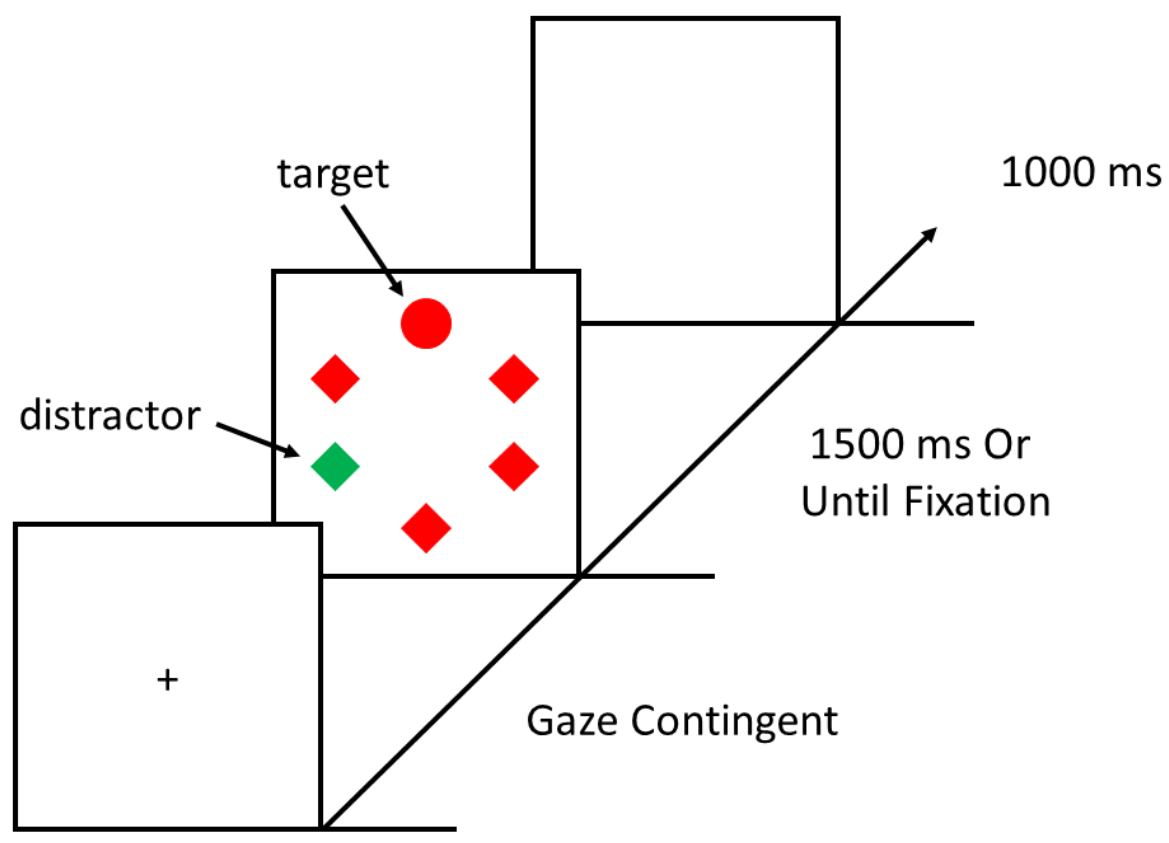

Figure 1. Sequence of trial events. The search array shows an example of a distractor-present trial.

visual angle) at the center of the screen. The fixation display remained on screen until eye position was registered within $1.1^{\circ}$ of the center of the fixation cross for a continuous period of 500 ms (as in Anderson \& Kim, 2019a, 2019b). The visual search array was then presented for $1500 \mathrm{~ms}$ or until a fixation on the target was registered. If a target was not fixated within the timeout limit, the words "Too Slow" would appear in the center of the screen for $1500 \mathrm{~ms}$. Lastly, the ITI consisted of a blank screen for 1000 ms. During the visual search task, participants had to search for the unique shape (one circle among diamonds, or vice versa). Each circle in the search array was $4.5^{\circ}$ visual angle in diameter and diamonds were $4.1^{\circ} \times 3.7^{\circ}$ visual angle. Each shape was placed at equal intervals along an imaginary circle with a radius of $10.2^{\circ}$. The color of the shapes was either red or green. In contrast to a prior study examining 
149 the influence of statistical learning of distractor location on oculomotor capture and sRT (Wang

150 et al., 2019a; see also Gaspelin et al., 2017), our task used gaze-contingent displays in which the 151 only action participants needed to perform was to fixate the target, with the task directly 152 probing eye movements. In the context of the influence of reward on attention, such an 153 approach produces a measure of oculomotor capture with high test-retest reliability (Anderson 154 \& Kim, 2019b) and the direct linking between eye movements and the requirements of the task 155 might facilitate a more sensitive test of the relationship between oculomotor capture and sRT

156 as a function of learning history. This approach is also more similar to studies that have

157 historically examined oculomotor capture as a function of sRT (e.g., Donk \& van Zoest, 2008;

158 Godjin \& Theeuwes, 2002; van Zoest et al., 2004; van Zoest \& Donk, 2005).

159 Design

Each participant completed 6 runs of 111 trials each (total 666 trials). The target was

161 present in each trial and a uniquely colored distractor singleton was presented in $67.6 \%$ of trials

162 (same shape as other non-targets but a different color). On distractor-absent trials, the target

163 position, shape, and color were fully counter-balanced. On distractor-present trials, the

164 distractor was in one position $2 / 3$ of the time (high-probability location) and equally often in all 165 other locations (thus, the distractor appeared in the high-probability location on $45 \%$ of all 166 trials). The high-probability location was counter-balanced across participants. The target 167 position was fully crossed in respect to the distractor position, and each target shape/color 168 combination was used equally-often over distractor-present trials. Trials were presented in a 169 random order. 


\section{Data Analysis}

Eye position was calibrated prior to each run using 9-point calibration and was manually drift corrected by the experimenter as necessary during the initial fixation display (as in Anderson \& Kim, 2019a, 2019b). During the presentation of the search array, the $X$ and $Y$ position of the eyes was continuously monitored in real time with respect to the six stimulus positions, such that fixations were coded online (as in Anderson \& Kim, 2019a, 2019b). The EyeLink 1000 Plus also exported an EDF file at the end of the experiment that contained detailed measurements concerning the saccades and fixations made on each trial in addition to markers for the beginning of each trial, presentation of visual search array, and the end of the trial for offline analysis. Fixation of a stimulus was registered if eye position remained within a region extending $0.7^{\circ}$ around the stimulus for a continuous period of at least $50 \mathrm{~ms}$ (100 ms on the target trigger the termination of the stimulus array; see, e.g., Anderson \& Kim, 2019a, 2019b). RT was measured from the onset of the stimulus array until a valid target fixation was registered. RTs in fixating the target that exceeded three standard deviations of the mean for a given condition for a given participant were trimmed (Anderson \& Kim, 2019b; Kim \& Anderson, 2020).

We measured which of the six shape stimuli was initially fixated on each trial (i.e., the first stimulus fixated) in addition to the time to fixate the target (i.e., response time, RT) from the eye data coded online. From this data, we calculated the proportion of initial fixations on the target and distractor when the distractor is in either a high-probability or low-probability location. Oculomotor dwell time on first fixations to the distractor (in high- and low-probability 
191 location) in addition to non-salient non-targets was computed as the average duration that the 192 eyes remained within the fixation window surrounding the stimulus, also from the eye data coded online. Lastly, saccadic RTs (sRTs) were computed as the time that the first saccade that landed outside of the fixation zone began relative to stimulus onset. This was computed offline using the EDF file. Saccades were defined as occurring when velocity exceeded $35^{\circ} / \mathrm{s}$ and acceleration exceeded $9,500^{\circ} / \mathrm{s}^{2}$ (Anderson \& Kim, 2018; Wang et al., 2019a). For each participant, we computed mean SRT for each distractor condition and additionally Vincintized distractor-present trials by sRT into 10 equally-sized bins (regardless of distractor condition), from which the proportion of oculomotor capture was computed for each of the two distractor conditions separately for each bin; this data was submitted to an analysis of variance (ANOVA)

201 with distractor condition (distractor at high-probability location, distractor at low-probability 202 location) and bin (1-10) as factors.

\section{Results}

\section{Time to Fixate the Target}

Time to fixate the target varied as a function of distractor condition (distractor at highprobability location, distractor at low-probability location, distractor absent), $F(2,70)=259.20$,

$207 p<0.001, \eta^{2}{ }_{p}=0.881$ (Figure 2A). A priori contrasts revealed that time to fixate the target was 208 slower for both of the distractor-present conditions relative to distractor-absent trials, 209 reflective of stimulus-driven attentional capture, $t s>8.65, p s<0.001, d_{z} s>1.44$, but was faster 210 for high-probability distractor trials compared to low-probability distractor trials, $t(35)=15.17$, $211 p<0.001, d_{z}=2.53$, reflective of statistically-learned distractor suppression. On distractor- 
212 absent trials, time to fixate the target was slower when the target appeared in the high-

213 probability distractor location compared to one of the other, low-probability distractor

214 locations, $t(35)=3.80, p=0.001, d_{z}=0.63$ (Figure $2 \mathrm{~B}$ ), also indicative of suppression.

Oculomotor Capture and Target Selection

Oculomotor capture was significantly less frequent when the distractor appeared in the

217 high-probability compared to one of the other, low-probability distractor locations, $t(35)=$

$21816.89, p<0.001, d_{z}=2.81$ (Figure $2 C$ ). This reduction in oculomotor capture came at the benefit

219 of initial fixations being on the target, which were correspondingly more frequent in the high-

220 probability distractor condition, $t(35)=16.82, p<0.001, d_{z}=2.78$ (Figure $2 C$ ). On distractor-

221 absent trials, the target was less likely to be the first stimulus fixated when appearing in the

222 high-probability distractor location, $t(35)=2.66, p=0.012, d_{z}=0.44$ (Figure 2D), also indicative

223 of location-specific inhibition.

\section{Distractor Dwell Time}

Dwell time on a non-target varied as a function of stimulus type (distractor at high-

226 probability location, distractor at low-probability location, non-salient non-target), $F(2,70)=$

$22739.84, p<0.001, \eta^{2} p=0.532$ (Figure 2E). Most critically, dwell time was shorter on high-

228 probability compared to low-probability distractors, $t(35)=6.59, p<0.001, d_{z}=1.10$, indicative

229 of accelerated distractor rejection. Both types of distractors were fixated longer than non-

230 salient non-targets, $t s>2.51, p s<0.018, d_{z} s>0.42$. 
A

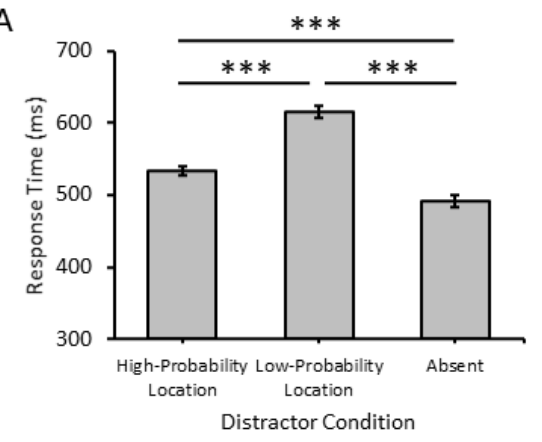

D

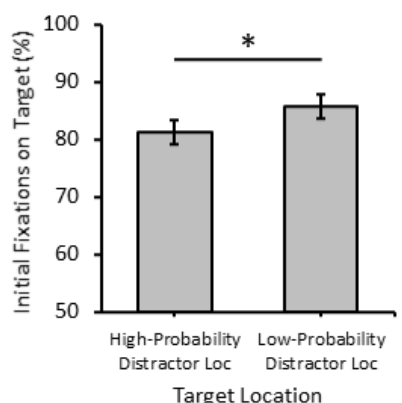

231

232

233

234

235

236

237

238

239

240

241

242

243

244

245

246
B

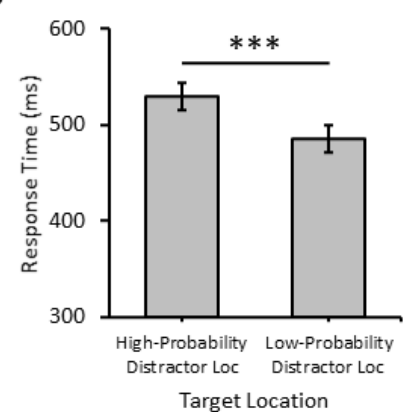

$\mathrm{E}$

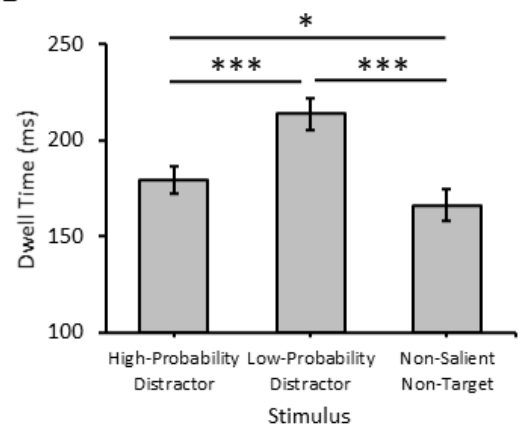

$\mathrm{C}$

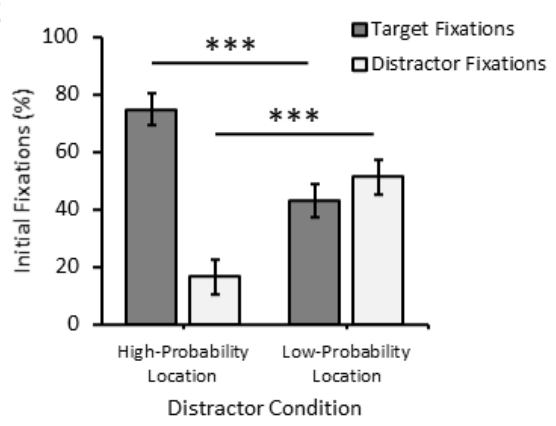

$\mathrm{F}$

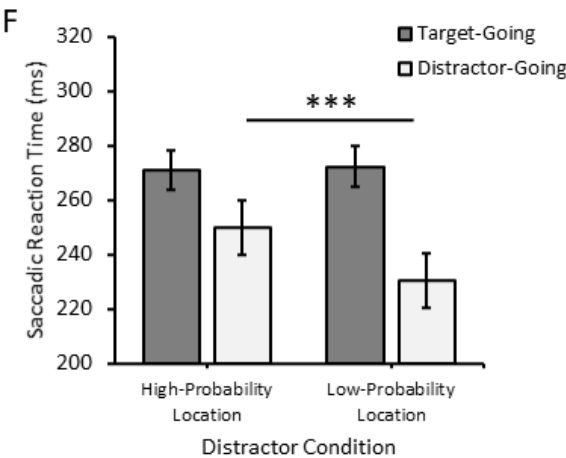

Figure 2. Behavioral results. Time to fixate the target (response time) on (A) distractor-present trials and (B) distractor-absent trials. (C) Percent initial fixations on targets and distractors as a function of the location of the distractor. (D) Initial fixations on the target on distractor-absent trials. (E) Dwell time as a function of the type of non-target. (F) Mean saccadic reaction time on distractor-present trials. Error bars depict within-subject confidence intervals calculated using the Cousineau method with a Morey correction. ${ }^{*} p<0.05$. ${ }^{* * *} p<0.001$.

\section{Relating Oculomotor Suppression to Saccadic Reaction Time (sRT)}

Distractor-present trials were separated into 10 equally-sized bins (Vincintized) based

on SRT regardless of distractor condition, such that the probability of oculomotor capture could be compared between the high- and low-probability distractor condition with SRT equated in each bin. There was a main effect of distractor condition that reiterates the effect collapsed over all trials described above, $F(1,35)=306.44, p<0.001, \eta^{2} p=0.897$ (Figure 3). There was a main effect of bin in which capture tended to decrease with increasing $\operatorname{sRT}, F(9,315)=32.79, p$ $<0.001, \eta_{p}^{2}=0.484$, reflecting the well-established relationship between oculomotor capture 
247 by physically salient stimuli and sRT (e.g., Donk \& van Zoest, 2008; Godjin \& Theeuwes, 2002;

248 van Zoest et al., 2004; van Zoest \& Donk, 2005). There was also a significant interaction

249 between distractor condition and bin, $F(9,315)=9.85, p<0.001, \eta^{2} p=0.220$, which was well-

250 accounted for by a linear trend with respect to the interaction term, $F(1,35)=26.78, p<0.001$,

$251 \quad \eta_{p}^{2}=0.433$, reflecting a strong dependence between SRT and capture for distractors in the low-

252 probability location while capture was generally infrequent across sRT for distractors appearing

253 in the high-probability location. Most importantly, a significant difference between distractor

254 conditions was evident for each of the ten bins, $t s>3.81, p s<0.002, d_{z} s>0.63$, including even

255 in the fastest bin in which eye movements tend to be most stimulus-driven, $t(35)=12.13, p<$

$2560.001, d_{z}=2.02$

We further examined mean SRT as a function of distractor condition to test whether the

258 time to initiate a saccade differed as a function of distractor condition. We first examined sRT

259 over all trials for each distractor condition, regardless of stimulus fixated (as in Wang et al.,

260 2019a), which revealed a slowing of sRT in the high-probability compared to the low-probability

261 distractor condition, $t(35)=4.38, p<0.001, d_{z}=0.73$. That is, even though participants were

262 overall significantly faster to fixate the target in the high-probability distractor condition, likely

263 due to substantially less frequent oculomotor capture by the distractor, the opposite was true

264 of how quickly they initiated an eye movement in this condition. Further analysis broke sRT

265 down additionally by whether the saccade was a target-going or distractor-going saccade. This

266 analysis revealed that the slowing of sRT on high-probability distractor trials was entirely

267 accounted for by a slowing of sRT for distractor-going saccades, $t(35)=4.72, p<0.001, d_{z}=$ 
0.79; SRT did not differ for target-going saccades, $t(35)=-0.26, p=0.799$ (Figure 2F). This

suggests that attentional priority for the distractor accumulated more slowly when appearing at

272 capture), it took significantly longer to reach that threshold.

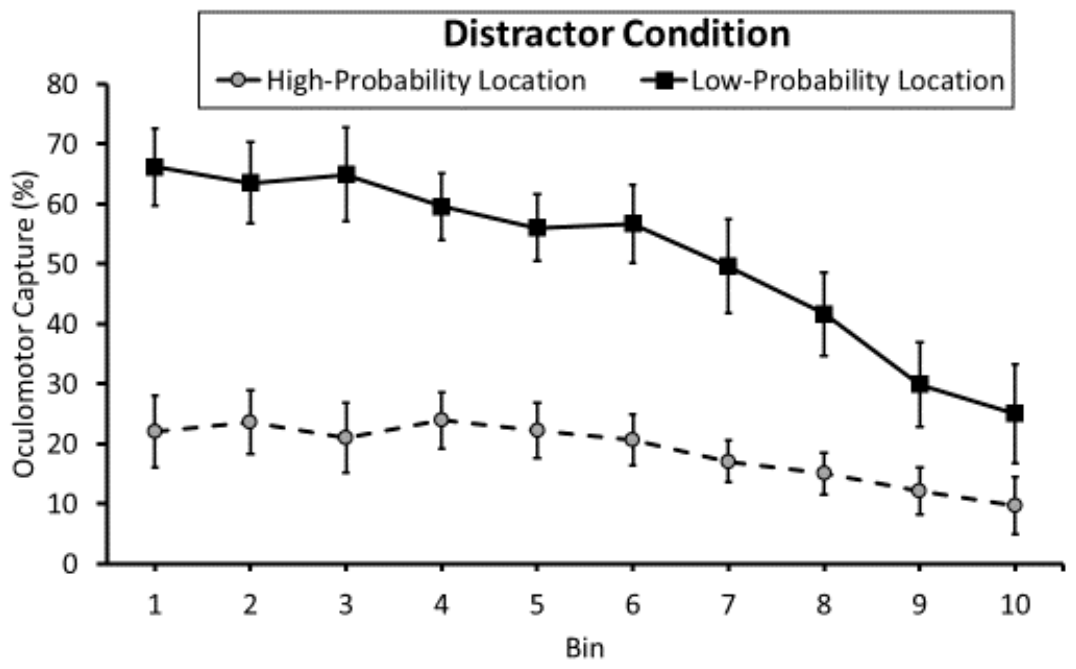

273

274

275

276

277

278

279
Figure 3. Oculomotor capture as a function of saccadic reaction time (sRT; Vincintized into 10 bins with bin 1 containing the fastest $10 \%$ of sRTs for each participant, bin 2 the next 10\%, etc.). Error bars depict within-subject confidence intervals calculated using the Cousineau method with a Morey correction.

\section{Examining the Role of Inter-trial Priming of Distractor Location}

All of the above analyses remain significant and essentially unchanged if all trials on which the position of the distractor immediately repeats from the prior trial are removed from analysis, consistent with prior research (Wang et al., 2019a), suggesting that our findings are not reducible to more frequent repetitions of distractor position in the high-probability distractor condition. 


\section{Examining the Speed with Which Effects of Statistical Learning on Distractor Suppression}

\section{Emerge}

To probe the speed with which the observed effects of statistical learning emerged, we performed the same analyses specifically on the first block of trials (with the exception of the analysis binning SRT since that analysis requires a large number of trials to represent each bin). All of the above-reported effects were significant when restricted to the first block of trials, with the exception of RT to fixate the target when appearing in the high-probability vs. a lowprobability distractor location, $t(35)=1.54, p=0.133$, and the proportion of first saccades on the target when comparing these same two conditions, $t(35)=0.66, p=0.512$; it is worth noting that in each of these two cases, the analysis relies on trials on which the target appears in the high-probability distractor location which occur infrequently, and so these particular analyses may be underpowered at the level of a single block of trials. Overall, the findings here suggest that the observed effects of statistically learned distractor suppression can emerge quickly, consistent with a prior report (Wang et al., 2019a).

\section{Discussion}

By examining oculomotor capture as a function of both SRT and the probability of a distractor appearing at a particular location, the present study provides novel insights into the mechanisms underlying statistically-learned distractor suppression. In combination with other oculomotor measures, we provide evidence for multiple mechanisms of distractor suppression working in tandem to support a reduction in the frequency of attentional capture as a function of statistical learning. Most strikingly, even the saccades that participants were the fastest to 
306 initiate were significantly less likely to be directed to the salient distractor, as if the salience of

307 the distractor was blunted as a result of statistical learning. Our findings here provide

308 straightforward behavior evidence of proactive distractor suppression, demonstrating a

309 reduced consequence of salience at the earliest stage of stimulus selection, confirming

310 interpretations of pre-trial EEG data using a similar paradigm (Wang et al., 2019b).

Replicating the well-established relationship between sRT and oculomotor capture (e.g.,

312 Donk \& van Zoest, 2008; Godjin \& Theeuwes, 2002; van Zoest et al., 2004; van Zoest \& Donk,

313 2005), saccades that were initiated more slowly were generally less susceptible to capture. It

314 was also the case that distractor-going saccades were slower to initiate when the distractor

315 appeared at the high-probability compared to a low-probability location. This suggests that

316 attentional priority accumulated more slowly for the distractor when appearing at the high-

317 probability location which, given with overall relationship between sRT and oculomotor

318 capture, may have served to further reduce the likelihood that a distractor at the high-

319 probability location would reach the threshold for triggering a saccade.

Replicating previous results (Wang et al., 2019a), we also found that participants were

321 faster to disengage attention from a distractor when appearing in a high-probability location.

322 This is a clear case of reactive attentional control, reflecting a shift in the speed with which a

323 stimulus can be rejected as a non-target. Although such an effect does not itself influence the

324 likelihood of attentional capture by the distractor, it mitigates the consequences of attentional

325 capture as a result of statistical learning. 
A significant effect of distractor probability on sRT was not observed in Wang et al.

327 (2019a), in contrast to the present study. However, in that study, a 10 ms difference collapsed

328 across distractor- and target-going saccades was observed in the same direction, which is

329 numerically quite similar to the significant $12 \mathrm{~ms}$ effect observed in the present study. With a

330 sample size more than twice as large in the present study, this apparent discrepancy may

331 simply reflect a consequence of reduced statistical power obscuring a comparatively smaller

332 effect, as indeed the study of Wang et al. (2019a) was powered to detect the much larger

333 consequence of distractor location probability on RT in target report (from Wang et al., 2018a).

334 The explicitly gaze-contingent aspect of our experimental design may have also resulted in

335 greater sensitivity to detect a link between SRT and oculomotor capture (see Methods).

336 Otherwise, we fully replicate the findings of Wang et al. (2019a), demonstrating highly robust

337 overall effects of statistical learning on oculomotor capture.

The present study lends important insights into why statistical learning can have such

339 substantial effects on the mitigation of distraction, and how distraction can be mitigated

340 through learning more generally. By providing straightforward evidence linking both proactive

341 and reactive mechanisms of distractor suppression to statistical learning in the same

342 experiment, our results demonstrate that multiple mechanisms are recruited to manage

343 distraction that are subject to learning from prior experience or selection history (see Anderson

344 et al., 2021; Awh et al., 2012). Our findings suggest that the reduction in distraction observed in 345 prior studies in which the probability of different distractor events is manipulated (e.g., Britton 346 \& Anderson, 2020; Kim \& Anderson, 2021; Wang \& Theeuwes, 2018a, 2018b, 2018c; Wang et 
347 al., 2019a, 2019b) reflects the sum total of multiple underlying mechanisms, with at least three 348 different time courses of information processing impacted. Attentional priority accumulates 349 more slowly for distractors appearing at the high-probability location, such that it takes longer 350 for a saccade to be initiated to the distractor in the event that it wins the competition for 351 oculomotor selection. In addition, for any given response speed, the attentional priority of the 352 distractor and its corresponding ability to compete for selection is overall reduced. These two 353 mechanisms result in a substantial reduction in the frequency with which distractors at high354 probability locations are fixated, producing facilitated visual search performance as reflected in 355 overall less time to localize the target. In the event that attention is still captured by the 356 distractor in spite of these learning-dependent changes, the speed of distractor rejection is 357 further subject to selection history, facilitating the disengagement of attention from more 358 frequently encountered distractor events and thereby further facilitating visual search 359 performance. Maximally efficient ignoring of task-irrelevant stimuli will optimize all three of these 361 aspects of attentional processing, which should be the focus of any learning procedure or 362 intervention designed to mitigate distraction. It does not appear to be the case that learning363 dependent ignoring can be reduced to a single consequence of learning on the attention 364 system, or likewise that only select components of the attention system are subject to learning 365 from selection history. Instead, our findings suggest that selection history reflects a systemic 366 shift in how information is processed on multiple levels. Future research should further explore 367 the scope and limits of such learning, in addition to how these different mechanisms of learned 
368 suppression are related to one another (e.g., Born et al., 2011). Our findings suggest that

369 learning to ignore is a multifaceted process, which should be more substantively taken into

370 account in the study of selection history.

371

373 Funding: This study was supported by NIH grant R01-DA046410 to BAA.

374 Conflicts of Interest Statement: The authors declare no conflict of interest.

\section{Author's Contributions:}

376 AJK and BAA contributed to the study concept and experiment design. AJK coded the

377 experiment oversaw data collection. AJK performed the data analyses under the supervision of 378 BAA. AJK and BAA interpreted the data and jointly contributed to the writing of the manuscript. 379 All authors approved the final version of the manuscript for submission.

\section{Open Practices Statement:}

382 The experiment reported in this article was not formally preregistered. Neither the data nor the 383 materials have been made available on a permanent third-party archive; requests for the data 384 or materials can be sent via email to the lead author at andyk@usc.edu. 


\section{References}

Anderson, B. A., \& Britton, M. K. (2020). On the automaticity of attentional orienting to threatening stimuli. Emotion, 20, 1109-1112.

Anderson, B. A., \& Kim, H. (2019a). On the relationship between value-driven and stimulusdriven attentional capture. Attention, Perception, and Psychophysics, 81, 607-613.

Anderson, B. A., \& Kim, H. (2019b). Test-retest reliability of value-driven attentional capture. Behavior Research Methods, 51, 720-726.

Anderson, B. A., \& Kim, H. (2018b). On the representational nature of value-driven spatial attentional biases. Journal of Neurophysiology, 120, 2654-2658.

Anderson, B. A., Kim, H., Kim, A. J., Liao, M.-R., Mrkonja, L., Clement, A., \& Grégoire, L. (2021). The past, present, and future of selection history. Neuroscience \& Biobehavioral Reviews, 130, 326-350.

Anderson, B. A., Laurent, P. A., \& Yantis, S. (2011). Value-driven attentional capture. Proceedings of the National Academy of Sciences, USA, 108, 10367-10371.

Awh, E. Belopolsky, A. V., \& Theeuwes, J. (2012). Top-down versus bottom-up attentional control: A failed theoretical dichotomy. Trends in Cognitive Sciences, 16, 437-443.

Born, S., Kerzel, D., \& Theeuwes, J. (2011). Evidence for a dissociation between the control of oculomotor capture and disengagement. Experimental Brain Research, 208, 621-631.

Brainard, D.H. (1997). The Psychophysics Toolbox, Spatial Vision, 10, 433-436. 
404 Britton, M. K., \& Anderson, B. A. (2020). Specificity and persistence of statistical learning in 405 406 distractor suppression. Journal of Experimental Psychology: Human Perception and Performance, 46, 324-334.

Donk, M., \& van Zoest, W. (2008). Effects of salience are short-lived. Psychological Science, 19, 733-739.

409 410
Failing, M., Feldmann-Wustefeld, T., Wang, B., Olivers, C., \& Theeuwes, J. (2019). Statistical regularities induce spatial as well as feature-specific suppression. Journal of Experimental Psychology: Human Perception and Performance, 45, 1291-1303.

Gaspelin, N., Leonard, C. J., \& Luck, S. J. (2017). Suppression of overt attentional capture by salient-but-irrelevant color singletons. Attention, Perception, and Psychophysics, 79, 4562.

Gaspelin, N., Leonard, C. J., \& Luck, S. J. (2015). Direct evidence for active suppression of salient-but-irrelevant sensory inputs. Psychological Science, 22, 1740-1750.

Geng, J. J. (2014). Attentional mechanisms of distractor suppression. Current Directions in Psychological Science, 23, 147-153.

Geng, J. J., \& DiQuattro, N. E. (2010). Attentional capture by a perceptually salient non-target facilitates target processing through inhibition and rapid rejection. Journal of Vision, 10(6):5, 1-12.

Godijn, R., \& Theeuwes, J. (2002). Programming of endogenous and exogenous saccades: Evidence for a competitive integration model. Journal of Experimental Psychology: Human Perception and Performance, 28, 1039-1054. 
425 Kim, A. J., \& Anderson, B. A. (2020). Threat reduces value-driven but not salience-driven attentional capture. Emotion, 20, 874-889.

427 Kim, H., \& Anderson, B. A. (2021). Combined influence of valence and statistical learning on the 428 control of attention: Evidence for independent sources of bias. Cognition, 208, 104554.

429 Kiss, M., Driver, J., \& Eimer, M. (2009). Reward priority of visual target singletons modulates event-related potential signatures of attentional selection. Psychological Science, 20, $245-251$.

Luck, S. J., Gaspelin, N., Folk, C. L., Remington, R. W., \& Theeuwes, J. (2021). Progress toward resolving the attentional capture debate. Visual Cognition, 29, 1-21.

434 Namian, M., Albert, A., \& Feng, J. (2018). Effect of distraction on hazard recognition and safety 435 risk perception. Journal of Construction Engineering and Management, 144(4), 04018008.

437 438

Schmidt, L. J., Belopolsky, A. V., \& Theeuwes, J. (2015). Attentional capture by signals of threat. Cognition and Emotion, 29, 687-694.

Stilwell, B. T., Bahle, B., \& Vecera, S. P. (2019). Feature-based statistical regularities of distractors modulate attentional capture. Journal of Experimental Psychology: Human Perception and Performance, 45, 419-433.

442 Strayer, D. L., \& Drews, F. A. (2004). Profiles in driver distraction: Effects of cell phone conversations on younger and older drivers. Human Factors, 46, 640-649.

444 Taneja, A., Fiore, V., \& Fischer, B. (2015). Cyber-slacking in the classroom: Potential for digital 445 distraction in the new age. Computers \& Education, 82, 141-151. 
446 Theeuwes, J. (2010). Top-down and bottom-up control of visual selection. Acta Psychologica,

447

448

449

450

451

452

453

454

455

456

457

458

459

460

461

462

463

464 $135,77-99$.

van Zoest, W., \& Donk, M. (2005). The effects of salience on saccadic target selection. Visual Cognition, 12, 353-375.

van Zoest, W., Donk, M., \& Theeuwes, J. (2004). The role of stimulus-driven and top-down control in saccadic visual selection. Journal of Experimental Psychology: Human Perception and Performance, 30, 746-759.

Vatterott, D. B., \& Vecera, S. P. (2012). Experience-dependent attentional tuning of distractor rejection. Psychonomic Bulletin and Review, 19, 871-878.

Wang, B., Samara, I., \& Theeuwes, J. (2019a). Statistical regularities bias overt attention. Attention, Perception, and Psychophysics, 81, 1813-1821.

Wang, B., \& Theeuwes, J. (2018a). Statistical regularities modulate attentional capture. Journal of Experimental Psychology: Human Perception and Performance, 44, $13-17$.

Wang, B., \& Theeuwes, J. (2018b). How to inhibit a distractor location? Statistical learning versus active, top-down suppression. Attention, Perception, and Psychophysics, 80, 860870.

Wang, B., \& Theeuwes, J. (2018c). Statistical regularities modulate attentional capture independent of search strategy. Attention, Perception, and Psychophysics, 80, 17631774. 
465 Wang, B., van Driel, J., Ort, E., \& Theeuwes, J. (2019b). Anticipatory distractor suppression elicited by statistical regularities in visual search. Journal of Cognitive Neuroscience, 31, 1535-1548.

468 Wolfe, J. M., \& Horowitz, T. S. (2017). Five factors that guide attention in visual search. Nature Human Behaviour, 1, 0058. 